\title{
Leukaemic stem cells go under the radar
}

PARP1
expression
by LSCs is
linked to
their selective
escape from
immune
surveillance by
NK cells

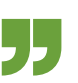

Treatment of acute myeloid leukaemia (AML) often fails and patients relapse because chemotherapy doesn't eliminate leukaemic stem cells (LSCs). A new study in Nature shows that LSCs not only selectively survive chemotherapy but also evade immune surveillance. LSCs are shown to lack expression of stress-induced ligands for NKG2D, which prevents the activation of cytotoxic lymphocytes such as natural killer (NK) cells.

Previous studies had noted variation in expression of NKG2D ligands (NKG2DLs) among cases of AML. So using an NKG2D-Fc chimeric protein, the authors screened 177 patients with AML for expression of NKG2DLs, which include the MIC and ULBP family proteins (MICA, MICB, ULBP1, ULBP2, ULBP5 and ULBP6). They found that even within individual patients there were subpopulations of $\mathrm{NKG}_{2} \mathrm{DL}^{+}$

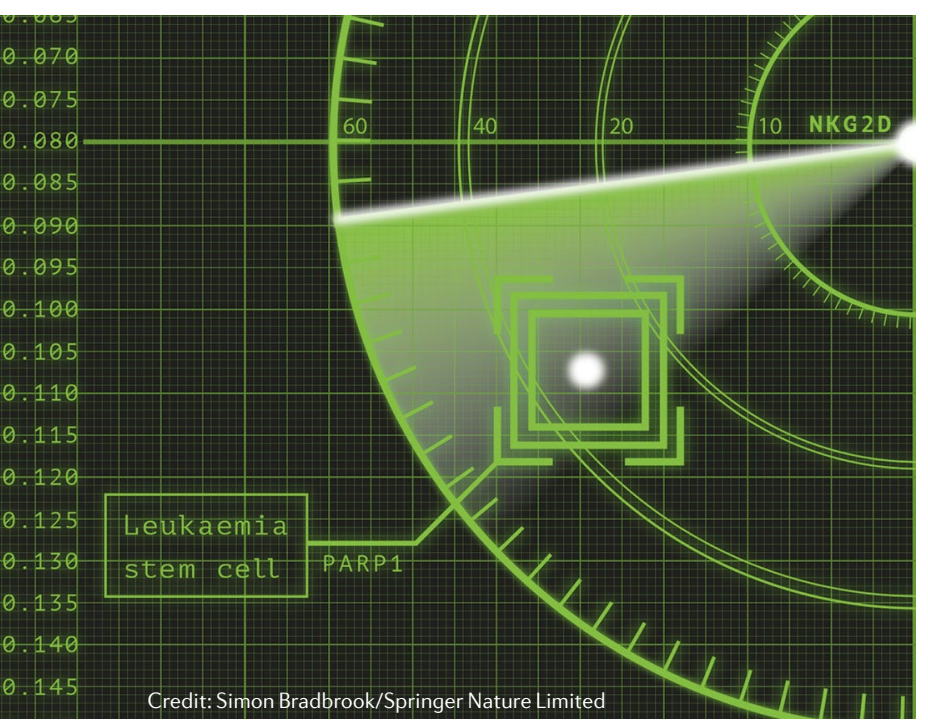

and NKG2DL- AML cells. NKG2DLcells had an immature morphology and a highly specific ability to repopulate immunodeficient mice following transfer.

In transplantation studies, human NKG2DL- AML cells infiltrated the mouse bone marrow more efficiently than NKG2DL ${ }^{+}$AML cells and were associated with poorer overall survival. Treatment of engrafted mice with a chemotherapy drug reduced the bone marrow infiltration of $\mathrm{NKG}_{2} \mathrm{DL}^{+}$but not NKG2DL- AML cells, indicating that NKG2DL- AML cells are more resistant to chemotherapy. Consistent with this finding, patients with higher proportions of $\mathrm{NKG}_{2} \mathrm{DL}^{+}$cells had a better response to chemotherapy and improved overall survival.

In support of a role for $\mathrm{NK}$ cells in the observed benefit from NKG2DL expression, co-cultures of bulk AML cells and polyclonal NK cells led to a preferential reduction of $\mathrm{NKG}_{2} \mathrm{DL}^{+}$cells; an effect that was abrogated by blockade of NKG2D. Moreover, transfer of AML cells from these NK cell co-cultures into immunodeficient mice led to faster engraftment and reduced survival compared with mice receiving AML cells from $\mathrm{NK}$ cell co-cultures containing NKG2D-blocking antibody. This indicates that NK cells kill non-stem leukaemic cells through an NKG2D-dependent mechanism, thereby enriching LSCs.

Further characterization of the subpopulations revealed that NKG2DL- cells had a gene signature associated with LSCs, including expression of stemness genes.
One of the 22 genes differentially expressed between the NKG2DLand $\mathrm{NKG}_{2} \mathrm{DL}^{+}$subpopulations encodes poly(ADP-ribose) polymerase 1 (PARP1), which is known to be involved in DNA damage responses. Higher expression of PARP1 by NKG2DL- cells was shown to repress the transcription of NKG2DLs, as genetic or pharmacological inhibition of PARP increased NKG2DL transcripts in bulk and NKG2DL- AMLs. Recruitment of PARP1 to MICA and $M I C B$ promoters confirmed a direct role for PARP1 in repression of NKG2DL genes.

Finally, the authors showed that transfer of AML cells pretreated with PARP1 inhibitor into immunodeficient mice together with NK cells led to a considerable reduction in tumour burden and improved survival compared with control settings, suggesting that PARP1 inhibition sensitizes AML cells to NK cell killing. Similarly, leukaemogenesis could be inhibited long-term by in vivo co-treatment of engrafted mice with the PARP1 inhibitor and human NK cells.

Together, these data describe how PARP1 expression by LSCs is linked to their selective escape from immune surveillance by NK cells. Besides a specific lack of expression of NKG2DLs, LSCs also show reduced expression of other NK cell activating ligands, such as DNAM1, of the costimulatory molecules CD80 and CD86 and of HLA class I molecules, suggesting that immune evasion in AML probably involves multiple mechanisms. Future experiments will address whether the immune-sensitizing effect of PARP inhibition may be a useful approach for treating AML in humans.

Lucy Bird

ORIGINAL ARTICLE Paczulla, A. M. et al. Absence of NKG2D ligands defines leukaemia stem cells and mediates their immune evasion. Nature https://doi.org/10.1038/s41586-0191410-1 (2019) 